\title{
A Unique Presentation of Posterior Reversible Encephalopathy Syndrome
}

\author{
Anupriya Saxena ${ }^{1}$, Ruchi Kapoor ${ }^{1}$, Ashok Kumar Saxena ${ }^{1}$
}

\section{Abstract}

Introduction: Posterior reversible encephalopathy syndrome is a neurologic condition which is often though not always is associated with Pregnancy induced Hypertension. A high index of suspicion in a pregnant patient who presents with seizure and altered sensorium, along with characteristic findings in magnetic resonance imaging which are pathognomic for this syndrome can help clinch the diagnosis. An early definitive diagnosis and prompt management is the key to prevent permanent neurological damage in the patient.

Case Report: We report a case of delayed unusual presentation (6 days post partum) of Posterior reversible encephalopathy syndrome [PRES] in a 25 year old pregnant patient of Pregnancy Induced Hypertension, wherein the patient presented six days after the delivery of baby in a unconscious state. She was admitted in intensive care unit and was managed with mechanical ventilation along with other neuroprotective measures. MRI brain was done which helped to clinch the diagnosis of PRES. She had complete recovery without any residual neurologic deficits and was discharged.

Conclusion: Prompt and early diagnosis and neuroprotective measures are the keys for an ideal management of Posterior Reversible Encephalopathy syndrome. Also MRI brain is essential to clinch the diagnosis.

Keywords: PRES, Pregnancy, Preeclampsia, Eclampsia.

\section{Introduction}

Posterior reversible encephalopathy syndrome (PRES) is a clinico-radiological syndrome with varied presentation. The symptoms may vary from headache, seizures, altered consciousness and visual disturbances to homonymous hemianopia and even cortical blindness. The patient may present with altered consciousness which could vary from mild confusion to agitation or even coma. The syndrome may or may not be associated with acute hypertension. Seizures and status epilepticus are common, however it is not pathognomic as non-convulsive status epilepticus has also been associated with PRES [1].

If recognized and treated early, the clinical syndrome commonly resolves within a week, thus early detection and confirmation of diagnosis is the key to management of PRES.

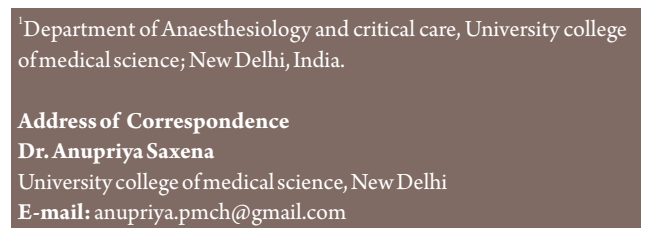

\section{Case Report}

A 25 year old gravid 2 para 2 live 1 patient presented to obstetric emergency our hospital with a history of 9 months amenorrhea, pain in lower abdomen and difficulty in breathing. On general physical examination patient was conscious and her GCS was $15 / 15$, pulse rate was 106 beats per minute, blood pressure was high 180/110, oxygen saturation $80 \%$ on room air, respiratory rate 22 per minute, and she had pink frothy secretion from the mouth along with generalised edema. Urine albumin ++ . On auscultation of chest bilateral basilar fine crepitations were heard, immediately the patient's trachea was intubated using Rapid sequence induction with injection Propofol and injection rocuronium, injection Xylocard $60 \mathrm{mg}$ along with it injection frusemide $20 \mathrm{mg}$ was given to manage pulmonary edema. The fetus was under distress with fetal heart rate of $90 /$ minute therefore the patient was taken for emergency caesarean section under general anaesthesia without waiting for an obstetric ultrasound. In the operating room anaesthesia was maintained on sevoflurane and oxygen achieving MAC 1, (nitrous oxide was avoided) and intermittent doses of rocuronium. The patient was ventilated with pressure control ventilation as the peak inspiratory pressure were high, peak pressure of $28 \mathrm{mmHg}$ before the delivery of baby and $22 \mathrm{~mm} \mathrm{Hg}$ after the delivery of baby. The oxygen saturation was maintained throughout at $100 \%$ and BP was high normal in the range of 130-140 mmhg systolic and 90-95 mmhg diastolic. The baby had delayed cry with a Apgar score of 6,8 and was shifted to Neonatal Intensive Care Unit for observation and further management. Following the birth of baby injection fentanyl 100 micrograms was administered. After the surgery neuromuscular blockade was not reversed and patient was shifted to ICU for further management.

In the ICU the further management of pulmonary edema was done with injection furosemide, injection morphine and positive pressure ventilation along with antibiotics and antihypertensives labetelol and magnesium sulphate were given as her blood pressure continued to be on higher side post surgery. The patient responded well to treatment, blood pressure was within normal limits and patient's trachea was extubated the next day. Magnesium sulphate was tapered and stopped, patient was shifted toward the next day. Later she was discharged from

(C) 2020 by Journal of Anaesthesia and Critical Care Case Reports|Available on www.jaccr.com | DOI: 10.13107/jaccr.2020.v06i01.150

This is an Open Access article distributed under the terms of the Creative Commons Attribution Non-Commercial License (http://creativecommons.org/licenses/by-nc/3.0) which permits unrestricted non-commercial use, distribution, and reproduction in any medium, provided the original work is properly cited. 
hospital after three days along with the baby. Three days after discharge that is sixth day postpartum, patient had one episode of generalized tonic-clonic seizure at home after which she came to gynecology emergency department in the evening hours in an unconscious state with a GCS of E2V1M2. On examination she was found to have an elevated BP of $180 / 110 \mathrm{~mm} \mathrm{Hg}$, pulse rate $94 /$ minute oxygen and saturation of $100 \%$ on room air. Immediately the patient trachea was intubated for airway protection after giving injection Xylocard, injection Thiopentone and injection Rocuronium in the emergency room. Physician opinion was sought and patient was administered loading dose of phenytoin and a bolus dose of injection labetalol as advised by physician. A provisional diagnosis of hypertensive emergency was made and the patient was then transferred to intensive unit for mechanical ventilation and further management. Subsequent morning after weaning from sedation neurologic assessment was done. Pupils were normally reactive to light and fundus examination was unremarkable. Cranial nerve examination, the motor power and sensory function could not be elicited as the patient was unconscious. Cerebellar signs were intact and there was no evidence of signs of meningeal irritations. Plantar reflexes were down going bilaterally.

Laboratory findings were suggestive of leucocytosis, total leucocyte count being 17000 , haemoglobin was $10 \mathrm{gm} \%$, remaining blood investigations were within normal limits. Urinalysis was remarkable for $3+$ protein. The coagulation and liver function tests were also within normal limits. The patient was managed with supportive treatment in form of antibiotics, tablet amlodipine $10 \mathrm{mg}$ OD through ryles tube and antiepleptics (phenytoin 100mg TDS) along with mannitol $100 \mathrm{ml}$ TDS, meanwhile a neurology consultation was sought.

On the advice of neurologist MRI BRAIN was done on third day post admission and it showed ill defined areas of T2W1 and FLAIR hyperintensities in right temporoparietal, bilateral occipital and bilateral high fronto parietal lobes in para saggital location, as well as bilateral ganglio thalamic regions showing partial restriction on DWI. These findings were suggestive of posterior reversible encephalopathy syndrome. Subsequently the treatment was reviewed and tablet acetazolamide $250 \mathrm{mg}$
QID was added for management of cerebral edema as advised by the neurologist.

While in the intensive care unit patient remained unconscious for initial 4 days and patients lungs were ventilated with SIMV mode of ventilation. It was only on 5th day that the patient started showing signs of neurologic recovery. Her consciousness level gradually improved, GCS score improved from initial of $5 / 15$ to finally $15 / 15$, weaning from mechanical ventilation was done and patient trachea was extubated on 7 th day in the ICU. Power was 5/5 in all the limbs, the sensory function was intact and patient had no cognitive impairment thus there was a complete neurologic recovery. The injection mannitol was discontinued and syrup glycerol 30ml TDS was started instead along with tablet phenytoin 100mg TDS and tablet acetazolamide $250 \mathrm{mg}$ QID. Patient was shifted then to a HDU on ninth day of admission and was discharged a week later. The subsequent course was unremarkable and patient was followed in the OPD for one month.

\section{Discussion}

Posterior reversible encephalopathy syndrome, also termed reversible posterior cerebral edema syndrome, affecting predominantly the white matter of the posterior cerebral hemispheres. PRES is a serious complication accompanying broad spectrum of clinical entities namely hypertension, preeclampsia/eclampsia and HELLP syndrome, hypertensive encephalopathy, nephropathy, use of immunosuppressive medication, systemic lupus, Henoch-Schonlein purpura and hemolytic uremic syndrome [2].

In the present patient there was sudden onset of seizure followed by unconsciousness accompanied with increased blood pressure which lead to the differential diagnosis of stroke, meningoencephalitis, PRES, demyelinatinglesion of brain, cerebrovenous thrombosis and hypertensive emergency with retinal haemorrhage. Retinal haemorrhage was excluded by fundus examination. Meningoencehephalitis was ruled out as the patient had no fever and no neck rigidity. Further the typical MRI findings and reversibility of symptoms clinched the final diagnosis of PRES.

In this case the presentation of PRES was unusual as the patient presented on sixth day postpartum after being discharged in a normal condition following an initial hospitalisation. Usually patients present with PRES within the initial $48 \mathrm{hrs}$ after delivery where as this patient presented so late that to after an uneventful stay at home following discharge. After through search in the existing literature we could not find any case report wherein the patient remained asymptomatic and presented this late to the hospital. In the ICU, though the patient remained unconscious for the initial 5 days but an early diagnosis and neuroprotective measures lead to complete neurologic recovery.

Multiple theories have been proposed to explain the pathophysiology. The more popular theory states that hypertension leads to failure of autoregulation leading to hyperperfusion, and vasogenic edema. The other theory suggests that vasoconstriction and hypoperfusion leads to brain ischemia and subsequent vasogenic edema [3].

Clinically, PRES presents with headache, seizures, encephalopathy, visual disturbances and focal neurological symptoms. As the name suggests, reversibility of these symptoms is one of the hallmarks of the disease. However delayed diagnosis and improper management can lead to irreversible sequela [4].

PRES has become better recognized with the progress made in imaging modalities. MRI is the imaging modality of choice to diagnose PRES. PRES appears as high signal intensity predominantly in the parietal and occipital region of the brain in T2 weighted scans. Diffusion-weighted MRI helps to distinguish the vasogenic oedema from cytotoxic oedema, which is characteristic of this disease [5].

Management of PRES is imperative to prevent development of complications such as permanent neurologic damage, coma. Maintaining normotension along with other neuroprotective measures are the cornerstones of the management of PRES. The patient like in this case may require ICU/HDU care and positive pressure ventilation for management depending on the severity of the underlying condition.

Conclusion and Clinical significance We have described a unusual presentation of PRES. A high index of suspicion, prompt and early diagnosis and neuroprotective measures are the keys for an ideal management of Posterior Reversible Encephalopathy syndrome. Also MRI brain is essential to clinch the diagnosis. 


\section{References}

1. Ural UM et al. Posterior Reversible Encephalopathy Syndrome in a Postpartum Preeclamptic Woman without Seizure. Case Reports in Obstetrics and Gynecology 2014; Article ID 657903.

2. Rijal JPetal.BMJ Case Rep 2014. doi:10.1136/bcr-2013-203162

3. Sodalaguntaa MB et al. Posterior Reversible Encephalopathy Syndrome.JNeurol Res.2016;6:106-110
4. Krupa BM, SundariN, MadhusudhanBK, SwaroopA, Chaitra S. Posterior reversible encephalopathy syndrome in pregnancy. J South Asain Feder Obst Gynae 2015;7(3): 205206.

5. Kumar N, Singh R, Sharma N, Jain A. Atypical presentation of posterior reversible encephalopathy syndrome: Two cases. JAnaesthesiol ClinPharmacol 2018;34:120-2.
Conflict of Interest: Nil

Source of Support: None

\section{How to Cite this Article}

Saxena A, Kapoor R, Saxena A K | A Unique Presentation of Posterior Reversible Encephalopathy Syndrome |Journal of Anaesthesia and Critical Care Case Reports|JanApril 2020; 6(1): 22-24. 